\title{
LUMINESCENT COMPOSITES ON THE BASE OF MICROCRYSTALLINE CELLULOSE: SYNTHESIS, FABRICATION AND PROPERTIES
}

\author{
Submited on 21/02/2019 - Accepted on 03/06/2019
}

\begin{abstract}
The data on preparation, structure and morphology of cellulose-based composites, those have oxide component as filler are reported. It is shown that depending on raw materials origin and processing degree cellulose samples reveal various degree of crystallinity (in the range $64-77 \%$ ). Obtained composites reveal even lesser crystallinity degree $\approx 57 \%$. Detailed analysis of surface morphology was performed with using of scanning electronic microscopy. It was found the studied samples contains plates with sizes $\sim 20-$ $50 \mu$ those consist of close-packed grains of 5-10 $\mu$ size. In the case of composites some oxide nanoparticles (sizes up to $200 \mathrm{~nm}$ ) were incorporated into grains of microcrystalline cellulose. It was established the luminescence properties of cellulose are dependent on type of plant raw materials as well as on processing methods. These factors have influence on luminescence intensity but profile of photoluminescence bands remains practically unchanged. Under excitation in 337-532 $\mathrm{nm}$ spectral region the composites that contain $\mathrm{K} 2 \mathrm{Eu}(\mathrm{PO} 4)(\mathrm{MoO} 4)$ and $\mathrm{LaVO} 4: \mathrm{Sm}$ are characterized by intensive visible photoluminescence. Spectra of these PL depend on excitation wavelength.
\end{abstract}

Keywords : microcrystalline cellulose ; composite ; oxide nanoparticles ; luminescence; dilatometry.

\author{
Y. NAOUI ${ }^{1}$ \\ S.G. NEDILKO ${ }^{2}$ \\ O.M. ALEKSEEV ${ }^{2}$ \\ YU.E. GRABOVSKYI ${ }^{2}$ \\ E.O. REZNICHENKO ${ }^{2}$ \\ V.P. SCHERBATSKYI ${ }^{2}$ \\ V.A. BARBASH ${ }^{3}$ \\ O.V. YASCHENKO ${ }^{3}$ \\ V.V. BOYKO ${ }^{4}$ \\ V.P. CHORNII 4 \\ M.S. NEDIELKO 5 \\ T. DORBANI ${ }^{1}$ \\ S. HAMAMDA 1 \\ 1 Laboratory TTSM, Frères Mentouri University \\ Constantine, Algeria. \\ 2 Taras Shevchenko National University of Kyiv. \\ Kyiv, Ukraine. \\ 3 National Technical University of Ukraine "Igor \\ Sikorsky Kyiv Polytechnic Institute", Kyiv, \\ Ukraine. \\ 4 National University of Life and Environmental \\ Sciences of Ukraine, Kyiv, Ukraine. \\ 5 Paton Electric Welding Institute of NAS of \\ Ukraine, Kyiv, Ukraine
}

\section{INTRODUCTION}

Cellulose, as one of the most common natural polymers on Earth, is widely used for the production of functional, and among them, nanocomposite photovoltaic materials and devices based on them. In particular, it can be sensors, drives, devices for flexible electronics, etc. [1 4]. However, cellulose and materials on its basis are not often used or studied as optical systems or systems where optical phenomena can be inquired. Up today, known examples of such applications are evidence of the promise of this development and applications direction. This applies, e.g, to the development of solar radiation dosimeters based on the composite structure consisting of an organic dye, titanium oxide ( $\mathrm{TiO} 2)$, as a photocatalyst, and a polyvinyl-pyrrolidone as a binding agent. These components are applied to the cellulose matrix (paper) by printing. Under influence of light, due to the photocatalytic reaction of titanium oxide, which is proportional to the duration of exposure and depending on the wavelength of light, there is a loss of color density of the dye. The level of color loss can be controlled on the eye, or instrumentally, by measuring the light coefficient reflection in ultraviolet (UV) or visible bands of solar radiation [5]. Cellulose fibers containing specific luminescent compounds can be effective modifiers for textile products, papers on paper and plastic. The essential advantage of oxide luminescent compounds as modifiers is their stability and the fact that it is difficult to remove them from the cellulosic fiber even using a special treatment [6-8].

The aim of the work is to develop micro/nanostructured composites based on microcrystalline cellulose (MCC) as matrix and some complex oxides as fillers, and to find out their structural and luminescent characteristics, which should confirm the perspectives of their practical application.

In this work, we give data on the procedure of synthesis, manufacturing and structural and optical (luminescent) characterization of cellulose and MCC, synthesized in laboratory conditions at the Department of Ecology and Technology of Plant Polymers Igor Sikorsky "KPI" University. These data are compared with the similar characteristics of commercial MCC and composite materials made on its basis by cold pressing $[9,10]$.

To achieve the goal of work it was necessary to solve a number of problems, namely: to make samples of MCC from different representatives of domestic plant raw materials and to study their structural, morphological characteristics and physical properties; to make composite structures on the basis of the MCC and complex oxides; to study their structure and morphology; to study the 
correlation between structural, morphological characteristics and physical properties of composites.

\section{MATERIALS AND METHODS OF STUDY :}

Several series of samples were investigated in this work. The A series, consisting of 5 specimens (A1, A2, .., A5), is a powdered material derived from a single plant material, the hemp fibers, at various stages of the synthesis procedure of the MCC. The B series, consisting of six samples (B1, B2, ..., B6), is an MCC, made from different representatives of domestic plants: flax fibers, Hemp, Kenafu, Straw Stems, Corn and Miskanthus. The method for the MCC obtaining samples is described in [11]. The commercial MCC tablets manufactured by ANCYR-B (Ukraine) were used as starting material for the production of $\mathrm{C}$ and $\mathrm{D}$ series samples of the samples. At first, the MCC tablets were spiked and dispersed using a rotary-planetary mill. Further, the identical parts of the resulting powder, weighing $\sim 0.5 \mathrm{~g}$, were mixed with a certain amount of pre-prepared oxide powder. Composite samples in the $\mathrm{C}$ series contained the nitrate agent, $\mathrm{AgNO}_{3}$. The design of the "MCC + AgNO3" composite, where $\mathrm{AgNO}_{3}$ was in amount $30 \mathrm{mg}$, is hereinafter referred to as $\mathrm{C} / \mathrm{Ag}$. The procedure for making samples of the D series was somewhat different from the previous one. Here a mixture of dispersed MCC and oxide was dissolved in $50 \mathrm{ml}$ of high purity ethanol and subjected to ultrasound treatment (frequency, $\mathrm{f}=4.2 \mathrm{kHz}$, processing time, $\mathrm{t}=20$ minutes). Then, the resulting suspension was kept for 30 minutes, the liquid merged and removed sediment filtered through a filter paper and dried in atmospheric environment at temperature of $60 \mathrm{C}$. Powders of mixtures of all series were compressed into tablets in the form of a disk with a diameter of $\sim 10 \mathrm{~mm}$ and a thickness of $\sim 1 \mathrm{~mm}$. The samples of $\mathrm{A}$ and $\mathrm{B}$ series were obtained at a pressure of $1.8 \times 10^{4} \mathrm{kPa} / \mathrm{m}^{2}$, and samples of $\mathrm{A}$ and $\mathrm{D}$ series - under pressure of $580 \mathrm{kPa} / \mathrm{m}^{2}$. The samples made of dispersed MCCs that do not contain oxide are designated as $\mathrm{O} / 0$, and all others: $\mathrm{D} / \mathrm{Name}$ of the appropriate oxide/Oxide content in $\mathrm{mg}$.

The wide range of non-destructive solid-state control methods was used for research, namely, X-ray diffraction analysis, optical and electron microscopy, luminescent spectroscopy and light reflection spectroscopy. The surface morphology of the manufactured samples was investigated using an optical microscope OLYMPUS GX51. A detailed view of the samples surface was obtained using Tescan Mira 3LMU electronic beam scanning electron microscope (SEM). During measurements the diameter of the beam was $20 \mathrm{~nm}$. Chemical elements microanalysis was performed using the instrumental tools of the same electron microscope. $\mathrm{X}$-ray diffraction analysis of the structure of the samples structure (XRD) was performed using a powder diffractometer DRON-3M, working in Bragg-Brentano $(\theta / 2 \theta)$ geometry and equipped with an X-ray tube BSV-28 (wavelength $\lambda_{\text {rad }}=1.54178 \AA$ ). The X-ray scattering spectra were recorded in the range of $10-70^{\circ} 2 \theta$ angles at the $0.1^{\circ}$ step. Characteristics of luminescent radiation and luminescence excitation spectra were investigated in UV and visible ranges of light using the laser-spectral complexes LSK-1 and LSK-2. With these complexes, the luminescent properties can be investigated in the wavelength range of excitation, $\lambda_{\mathrm{ex}}, 225-800 \mathrm{~nm}$, and in the range of wavelengths of the radiation registration, $\lambda_{\text {reg }}$, $300-1200 \mathrm{~nm}$.

\section{RESULTS AND DISCUSSION}

\section{A. Analysis of Optical and Electron Microscopy Data :}

Optical microscopic images show that the surface and, apparently, the volume of samples, as "pure" - without oxides, and incorporated by them, are rather heterogeneous.

The different brightness of the image parts indicates that they are plates (plateau), located at different depths and under slightly different angles to the surface (Fig. 1 left part). A detailed analysis of the surface performed by SEM shows that these plates have a size $\sim 20-50$ microns, and they themselves consist of tightly packed grains in the size of 5 - 10 microns. The said plateau can in some cases be separated by noticeable cracks (Fig. 1 right part). In addition to the details described, it's easy to see the inclusion of another type - they have excellent color, shape and size. Obviously, these inclusions are particles of oxide compounds embedded into the matrix of the MCC. The size of the particles lies between $10-200$ $\mathrm{nm}$. We also see that oxide nanoparticles are capable of, partially agglomerating in grain size 2 - 10 microns. Observed features of morphology of samples allow us to qualify the structure of pressed samples as similar to ceramic [9].

\section{B. Qualitative and quantitative analysis of the manufactured samples composition:}

The analysis of the manufactured samples composition was performed in different zones of the investigated samples. Some of them in Fig. 1b is marked with colored rectangles $1-3$. It is not surprising that the average content of carbon atoms, $\mathrm{C}$, and oxygen, $\mathrm{O}$, for all samples in the zones of the above plates (zone 1) is 7476 and $23-25 \%$, respectively. These data confirm the above conclusion that observed in SEM images plates are the blocks of the matrix of the MCC.
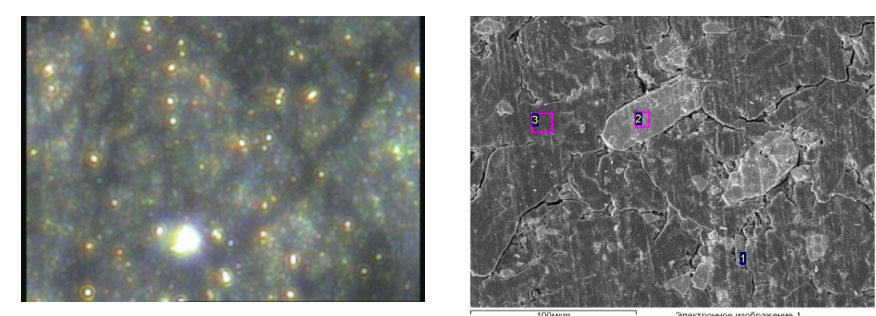

Fig. 1. Optical (left) and SEM (right) images of the $\mathrm{D} / \mathrm{K}_{2} \mathrm{Eu}\left(\mathrm{PO}_{4}\right)\left(\mathrm{MoO}_{4}\right) / 100$ sample 
The analysis of the mentioned inclusions (grains and their agglomerates) in composite samples showed that they really belong to the corresponding oxides, because their composition corresponds to the chemical composition of the oxides used [9].

\section{XRD data analysis :}

X-ray diffraction spectra of the MCC investigated samples in the range of $10-45^{\circ} 2 \theta$ angles demonstrate a series of diffraction lines associated with the diffraction of $\mathrm{X}$-rays on the cellulose matrix. These relatively broad lines are approximately located at $2 \theta$ angles $16 ; 22.5$ and $34.5^{\circ}$. For all samples, lines and shoulders of lower intensity are also observed (Fig. 2).

There are additional lines for composite samples. Their set, position and intensity distribution correspond to X-ray diffraction spectra of oxide compounds incorporated into composites. As the content of oxides increases, the intensity of these lines increases. Thus, it can be argued that these lines correspond to the inclusion of micro/nanoparticles of oxides to the cellulosic matrix, which is confirmed by comparison of these lines characteristics (position and intensity distribution) with the corresponding characteristics for $\mathrm{AgNO}_{3}, \mathrm{La}_{1}$ ${ }_{\mathrm{x}} \mathrm{RE}_{\mathrm{x}} \mathrm{VO}_{4}$ and $\mathrm{K}_{2} \mathrm{Eu}\left(\mathrm{PO}_{4}\right)\left(\mathrm{MoO}_{4}\right)$ compounds [9]. The diffraction spectra of the of the MCC matrix both by the number of lines, their position and intensity distribution are similar for all investigated samples - "pure" and those containing oxide. At the same time, more detailed analysis shows some differences. So, firstly, the halfwidth of the lines is somewhat dependent on the origin of the samples: laboratory (series A and B) or commercial (series C and D), including the presence of oxide (series $\mathrm{C}$ and D) in their composition. These facts mean that as a procedure for removing the crystalline phase of cellulose from the starting material, which is different for laboratory and commercial specimens, and the type of oxide and its amount in the matrix, affect the distribution of nanosized MCC particles by size.

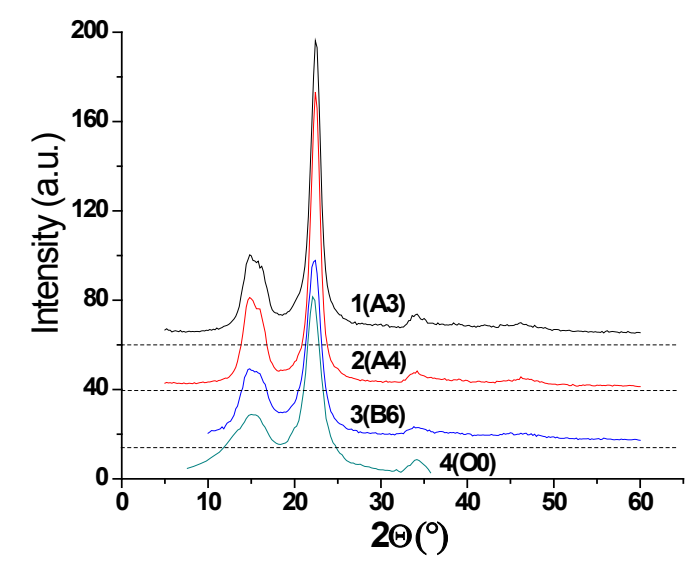

Fig 2 : X-ray diffraction spectra for samples of different stages of MCC preparation $(1,2)$ and for finished MCC $(3,4) ; 3$ laboratory, 4 - commercial MCC. (The sample number in the series is shown in the picture).
On the basis of the measured X-ray diffraction spectra, we also estimated the crystallinity of the samples $k$, which characterizes the relative content of the crystalline phase in the investigated material. For this, the contribution of the amorphous phase $\left(\mathrm{I}_{\mathrm{am}}\right)$ has been removed from the general spectrum: $\left(I_{\text {cryst }}+I_{a m}\right)$. Here $I_{c r}$ is the area in the spectrum under the lines corresponding to the diffraction on the crystalline structure of the cellulose matrix, and $I_{a m}$ is the area of the continuous background on which the lines are located, and this background is due to scattering on the amorphous composite material. The method of the crystalline and amorphous component in the scattering spectra separating is shown in Fig. 2. (Details of this procedure can be found in [9].) Thus, the degree of crystallinity $\mathrm{k}$ was estimated by the formula:

$$
\kappa=\frac{I_{c r}}{I_{c r}+I_{a m}}
$$

Calculated values fall within the limits of 64-77\% and 61-66 \% for "pure" samples of laboratory and commercial $\mathrm{MCC}$, respectively, whereas for composites the values of crystallinity $\mathrm{k}$ are significantly lower and make up 57 $58 \%$. Thus, the data on crystallinity indicate, firstly, the influence of the MCC synthesis procedure on the distribution of the crystalline and amorphous phases of cellulose in the finished product - MCC, and secondly, they show the influence of oxide particles on the structure and morphology of the crystalline phase of the MCC. The role of oxide micro/nanoparticles, obviously, is manifested in some destruction of the cellulose crystal lattice.

\section{Dilatometric behavior of the MCC and composites.}

Discussing the results of dilatometric behavior of the samples under study $\left(\Delta \mathrm{L} / \mathrm{L}_{0}\right.$ dependences, where $\Delta \mathrm{L}=$ $\mathrm{L}(\mathrm{T})-\mathrm{L}_{0}$ is the change of the sample size after heating from starting $\mathrm{T}_{0}$ temperature to temperature $\mathrm{T}$ ), it is easy to observe similar variation in dimension for the all composite samples, while $\Delta \mathrm{L} / \mathrm{L}_{0}$ behavior of the "pure" sample differs radically. Moreover, variations of the $\Delta \mathrm{L} / \mathrm{L}_{0}$ values are in the same range, despite of different behavior of corresponding $\Delta \mathrm{L} / \mathrm{L}_{0}$ curves. This range is near $13-2310^{-3}$, while $\Delta \mathrm{L} / \mathrm{L}_{0}$ reaches value of $4010^{-3}$ for the sample of minimal amount of oxide component (Fig. 3).

The description made above coincides with the data about coefficient of thermal expansions, $\alpha(\mathrm{T})$. We have found that $\alpha(T)$ values varies differently with temperature and the difference in variation is significant for the "pure" sample and "MCC-oxide" composites. The hidden peak of the $\alpha(\mathrm{T})$ curve is below of $40{ }^{\circ} \mathrm{C}$ and two strong dilatometric singularities are near 70 and $90{ }^{\circ} \mathrm{C}$ for the "pure" sample. Similar three features form $\alpha(\mathrm{T})$ curve for the sample of lowest oxide amount, we suppose, but they are smeared and shifted to the high temperature side, if compare with "pure" sample. (Their possible positions are near 35,80 , and $100{ }^{\circ} \mathrm{C}$.) When oxide amount increases, only one singularity is seen near 92 and $97^{\circ} \mathrm{C}$ on the $\alpha(\mathrm{T})$ 
curves (Fig. 4). Described peculiarities imply that the mentioned "pure" and composite samples are strongly different. This fact is related with impact of oxide particles on the structure, morphology and size of the cellulose matrix.

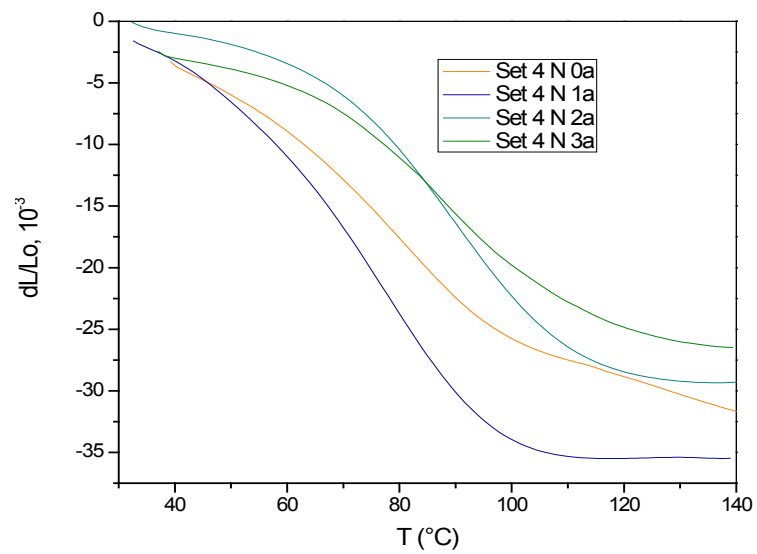

(a)

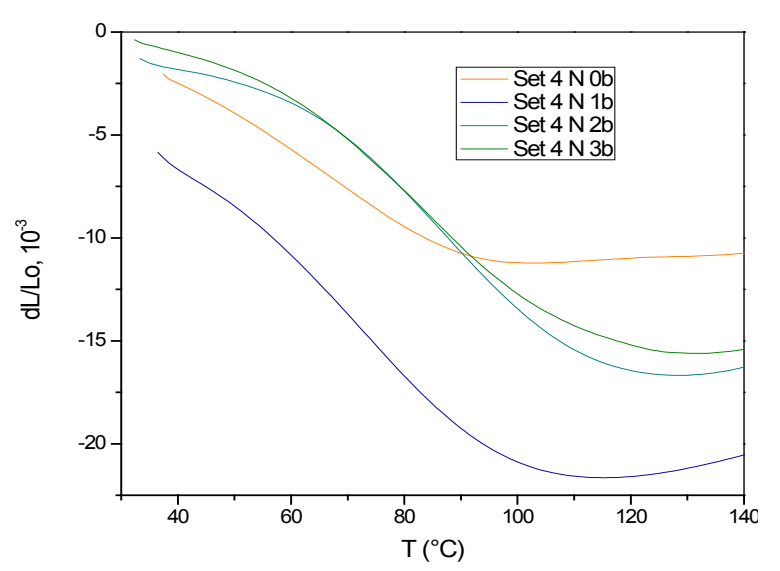

(b)

Fig. 3. dL/L for samples of different stages of MCC preparation $(1,2)$ and for finished MCC $(3,4) ; 3$ - laboratory, 4 commercial MCC. (The sample number in the series is shown in the picture).

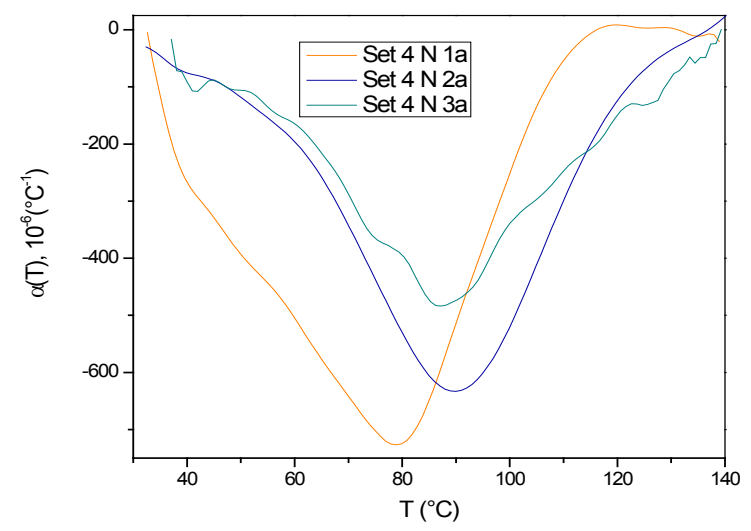

(a)

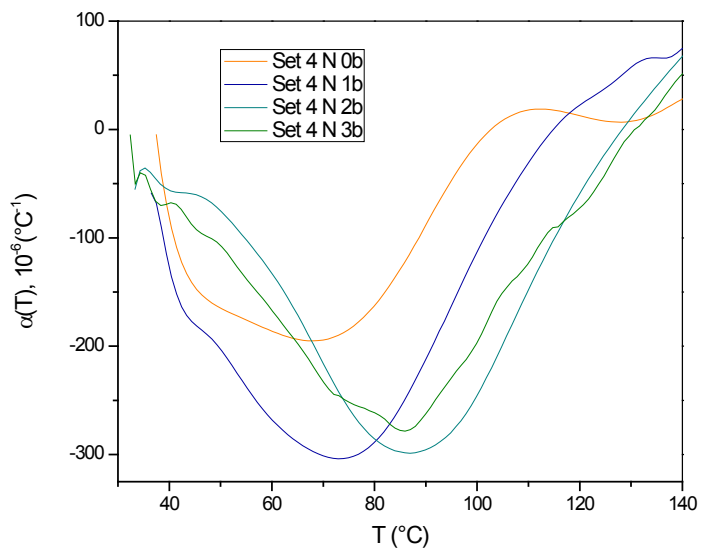

(b)

Fig. 4. Coefficient of dilatation for samples of different stages of MCC preparation $(1,2)$ and for finished MCC $(3,4) ; 3$ -

laboratory, 4 - commercial MCC. (The sample number in the series is shown in the picture).

\section{E. Fluorescent properties of MCC and composites:}

A wide structural band is observed in the PL spectra of MCC produced by us at UV and violet light excitation $\left(\lambda_{\text {ex }}=325-450 \mathrm{~nm}\right)$ regardless of MCC origin and prehistory. In the range of visible light, in this band, at least three rather intensive components that lie within the limits of 375-500, 450-550, 525-625 and 625-725 nm (can be distinguished (Fig. 5). The most intensive is the component of blue-green radiation, $450-550 \mathrm{~nm}$, within which lies the maximum of the spectral band of PL, $\lambda_{\max }$ $=\sim 490 \mathrm{~nm}$, at its excitation with $\lambda_{\text {ex }}=405 \mathrm{~nm}$. The PL spectra of the samples at the stages of MCC preparation and spectra of the "pure" MCC show that the pre-history of the samples and therefore their composition, structure and morphology are in some way reflected in the characteristics of the luminescence. The oxide filler also plays some role and the difference of curve 5 in Fig. 3, which corresponds to the radiation of the "MCC+ $\mathrm{AgNO}_{3}$ " composite, from the spectra of pure" MCC (Fig. 5 , curves 3,4 ) confirms that.

Therefore, we have the opportunity to assert that the observed radiation is associated with luminescent processes in the MCC matrix itself. Unfortunately, the question of their origin and the role of specific molecular components of the MCC or its derivatives (for example, carbonyl groups) is not fully understood. At the same time, the role in the luminescent processes of some molecular radicals, for example, hydroxyl groups, located on the surface of cellulose fibers, has previously been discussed.

The MCC+oxide" composites " in the case when the oxide compound in its composition contains luminescent active centers such as ions of rare earth elements $\left(\mathrm{Eu}^{3+}\right.$, $\mathrm{Sm}^{3+}$, etc.), are characterized by bright luminescence when excited by UV or visible radiation. The PL spectra of such composite samples essentially depend on the 
wavelength of excitation (Fig. 6). Thus, the blue-green radiation which is characteristic for the above-described emission of the cellulose matrix, dominates in the PL spectra at short-wave excitation $\left(\lambda_{\mathrm{ex}}=325-405 \mathrm{~nm}\right)$.

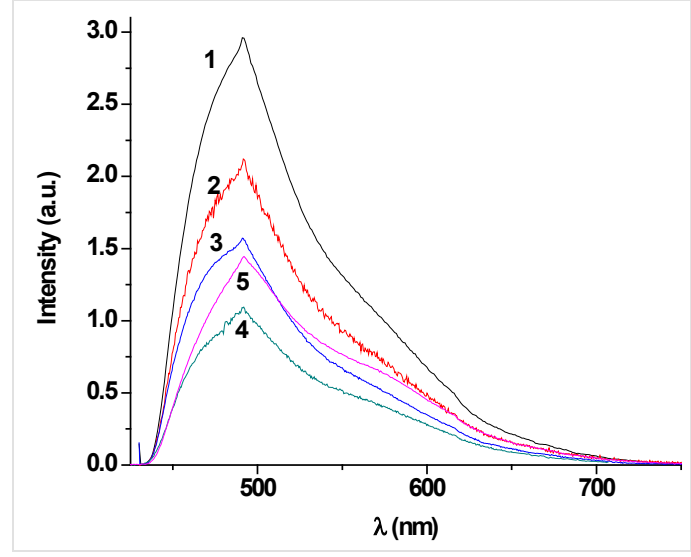

Fig.5. The PL spectra of the samples: A2 (1), A3 (2), A5 (3), $\mathrm{B} 6$ (4), and $\mathrm{C} / \mathrm{AgNO}_{3}$ composite (5)
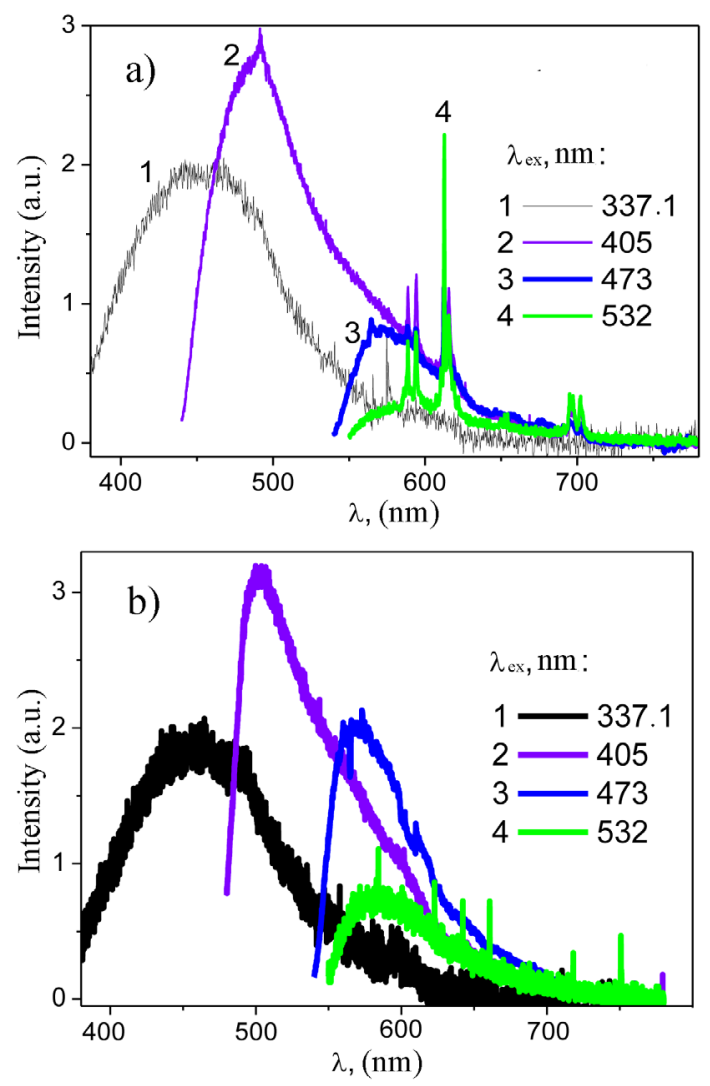

Fig. 6. The PL spectra of the composites $\mathrm{D} / \mathrm{K} 2 \mathrm{Bi}(\mathrm{PO} 4)(\mathrm{MoO} 4): \mathrm{Eu} / 100$ (a) and D/LaVO $4: \mathrm{Sm}: \mathrm{Eu} / 10$ (b); the wavelength of excitation is indicated in the Figure; $\mathrm{T}=300$ $\mathrm{K}$.

The use of longer-wave excitation leads to an increase in the intensity of the long-wave components of the PL and the displacement of the maximum of the PL band to the long-wavelength side of the spectrum and to the appearance of line spectra which is characteristic of rear - earth ions, $\mathrm{Eu}^{3+}$ and Samarium, $\mathrm{Sm}^{3+}$, in solid-state matrices [9].

\section{CONCLUSIONS}

1. Composite samples made by cold pressing method, where microcrystalline cellulose (MCC) is a matrix and the complex oxide compounds $\mathrm{AgNO}_{3}, \mathrm{LaVO}_{4}: \mathrm{Sm}, \mathrm{Eu}$ and $\mathrm{K}_{2} \mathrm{Bi}\left(\mathrm{PO}_{4}\right)\left(\mathrm{MoO}_{4}\right)$ : $\mathrm{Eu}$ are the fillers, have a ceramiclike structure formed by the MCC plates and oxide grains.

2. The composites are characterized by intense photoluminescence in visible light, whose spectrum consists of a wide band of luminescence of the cellulose matrix and a set of narrow lines caused by the emission of ions of rare-earth elements: $\mathrm{Eu}^{3+}$ and samarium, $\mathrm{Sm}^{3+}$.

3. Taken results are the basis for further development of the studied cellulose-oxide composites as violet and blue light luminescent transformers and followed creation on their basis of new type of the "white" light emitting diodes.

\section{REFERENCES}

[1] W.W. Tassi, G Nancy, Z. Hongl, "Nanocellulose-based translucent diffuser for optoelectronic device applications with dramatic improvement of light coupling," Applied materials and interfaces, vol. 7, pp. 26860 - 26864, 2015.

[2] X Wang, K.Z. Gao, Z.Q. Shao, X.Q. Peng, X. Wu, F.J. Wang, "Layer-by-layer assembled hybrid multilayer thin film electrodes based on transparent cellulose nanofibres paper for flexible supercapacitors applications," J. Power Sources, vol. 245 pp.148$155,2014$.

[3] A.C.M. Moraes, P.F. Andrade, A.F. Faria, M.B. Simoes, F.C.C.S. Salomao, E.B. Barros, M.D. Goncalves, O.L. Alves, "Fabrication of transparent and ultraviolet shielding composite films based on graphene oxide and cellulose acetate," Carbohydr. Polym., vol. 123, pp. 217-227, 2015.

[4] H.Z. Song, L.W. Zheng, "Nanocomposte films based on cellulose reinforced with nano- $\mathrm{SiO}_{2}$ : microstructure, hydrophilicity, thermal stability, and mechanical properties," Cellulose, vol. 20, pp. 1737 1746, 2013.

[5] P.S. Khiabani, A.H. Soeriyadi, P.J. Reece, J.J. Gooding, "PaperBased Sensor for Monitoring Sun Exposure," ACS Sens., vol 1, pp. 775-780, 2016.

[6] Yu Bian, Beihai He, Junrong Li, "Preparation of cellulose-based fluorescent materials using Zinc sulphide quantum dot-decorated graphene by a one-step hydrothermal method," Cellulose, vol. 23 , pp. 2363-2373, 2016.

[7] P. Kulpinski, A. Erdman, T. Grzyb, S. Lis, "Luminescent cellulose fibers modified with cerium fluoride doped with terbium particles." Polymer composites, vol 37, pp. 153-160, 2016.

[8] T.G. Rials, W.G. Glasser, "Thermal and dynamic mechanical properties of hydroxypropyl cellulose Films," J. Appl. Polym. Science., vol. 36, pp.749-758, 1988.

[9] M. Nedielko, S. Hamamda, O. Alekseev, V. Chornii, M. Dashevskii, M. Lazarenko, K. Kovalov, S.G. Nedilko, S. Tkachov, S. Revo, V. Scherbatskyi, "Mechanical, dielectric, and spectroscopic characteristics of "micro/nanocellulose + oxide" composites," Nanoscale Research Letters, vol. 12, pp. 98, 2017.

[10] V.A. Barbash, M.G. Karakutsa., I.V. Trembus, O.V. Yaschenko, "Development of technology of microcrystalline cellulose from hemp fibres," Eastern-European J. of Enterprise Tech., vol. 3/6, pp. 51-56, 2016

[11] V.A. Barbash, Yu.M. Nagorna, "Technology of the microcrystalline cellulose production from non-wood vegetable raw materials (in Ukrainian)," Naukovi visti NTU "KPI"», vol 2, pp. 119-124, 2015. 